\title{
Mapeamento da área Redes Sociais a partir da Web of Science
}

\author{
Alexandre D. Alves' ${ }^{1}$, Horacio H. Yanasse ${ }^{2}$, Nei Y. Soma ${ }^{1}$ \\ ${ }^{1}$ Instituto Tecnológico de Aeronáutica (ITA) \\ Centro Técnico Aeroespacial (CTA) - 12.228-090 - São José dos Campos - SP - Brasil \\ ${ }^{2}$ Universidade Federal de São Paulo (UNIFESP) \\ Rua Talim, 330 - Vila Nair - 12.231-280 - São José dos Campos - SP - Brasil \\ alexdonizeti@gmail.com, horacio.yanasse@unifesp.br, soma@ita.br
}

\begin{abstract}
Social networking is currently an area of great interest from researchers worldwide. This becomes apparent by the significant increase in the number of articles published in journals in the Web of Science in recent years. This paper aims to present a mapping of scientific production in the field Social Networks considering only the articles published in journals indexed in the Web of Science. To accomplish this we carried out a bibliometric analysis and an analysis of co-authorship networks to main countries and authors of the field. The result shows that not always do the countries and authors who publish more play the major role in the area.
\end{abstract}

Resumo. Redes Sociais é atualmente uma área de grande interesse por parte dos pesquisadores em nível mundial. Isso pode ser evidenciado pelo aumento significativo do número de artigos publicados em periódicos na Web of Science nos últimos anos. O objetivo deste trabalho é apresentar um mapeamento da produção cientifica da área Redes Sociais considerando os artigos publicados em periódicos indexados na Web of Science. Para isso foi realizada uma análise bibliométrica e uma análise das redes de coautoria para os principais países e autores da área. O resultado mostra que nem sempre os países e autores que mais publicam são os que mais influenciam a esta área.

\section{Introdução}

Redes Sociais é atualmente uma área de grande interesse por parte dos pesquisadores em nível mundial. Borgatti et al. (2009) já afirmavam isso, pois na década passada o número de artigos quase triplicou na Web of Science (WoS).

O objetivo deste trabalho é apresentar um mapeamento da produção científica da área Redes Sociais considerando informações sobre os artigos publicados em periódicos que estão indexados na WoS. O mapeamento de uma área permite contar parte de sua própria história, e ainda, pode indicar o perfil da colaboração científica nas publicações dos pesquisadores. Assim, é possível identificar àqueles que têm contribuído de forma significativa para uma determinada área. Nesta direção foram, então, realizadas análises bibliométrica e das redes de coautoria para países e autores da área Redes Sociais que mais publicaram em periódicos na WoS, incluindo distribuição geográfica da autoria, análise temporal dos periódicos e palavras-chave mais utilizadas e das medidas de centralidade.

A principal contribuição deste trabalho é mostrar que estudos mais abrangentes podem ser realizados, não somente variando os tipos de análises, mas também na 
quantidade dos dados a ser analisada. Percebe-se que em muitos trabalhos o número de artigos considerados nas análises é muito pequeno. Provavelmente, isso pode indicar a inadequação das ferramentas utilizadas para a coleta, extração e indexação desses dados.

Este artigo está organizado da seguinte maneira: Na Seção 2 é apresentada uma breve descrição sobre Redes Sociais, destacando os principais conceitos sobre Análise de Redes Sociais, visando o entendimento de termos utilizados neste trabalho. Também são apresentados alguns trabalhos relacionados, destacando o que foi considerado para a realização deste trabalho. Na Seção 3 é descrito como foram coletados os dados de interesse e listada as ferramentas utilizadas. Na Seção 4 são apresentados os resultados, destacando as principais informações obtidas. Finalmente, na Seção 5 são apresentadas as conclusões.

\section{Principais conceitos e trabalhos relacionados}

Uma rede social é composta por um conjunto de atores e relações definidas entre esses [Wasserman e Faust 1994]. Um ator pode representar um indivíduo (e.g., um pesquisador ou uma instituição) ou um grupo (e.g., instituições federais de ensino do país). As relações são dadas por vínculos (e.g., profissional ou familiar) e podem ser direcionais ou não.

Redes sociais podem ser representadas por meio de sociogramas, que são grafos com vértices representando atores e arestas as relações entre esses. O estudo de redes através de grafos remonta a Euler quando da solução do famoso problema das pontes de Königsberg, a qual originou a "teoria dos grafos" [Newman et. al 2006]. O estudo de redes sociais foi iniciado em meados de 1930 por Moreno (1934), quando sociólogos utilizavam redes para estudar o comportamento da sociedade e a relação entre as pessoas. Um estudo importante nesta área foi o de Milgram [Milgran 1967] através de suas experiências que levaram ao conceito de "mundo pequeno" (small world). Watts e Strogatz (1998) tentaram comprovar que as pessoas estão separadas por seis graus (no máximo), ou seja, pessoas aparentemente sem relação alguma têm grande probabilidade de possuírem, em algum grau, amigos em comum que as aproximem.

A partir desses trabalhos, desenvolveu-se um novo método de análise denominado "Análise de Redes Sociais - ARS" (Social Network Analysis - SNA). A ARS é uma abordagem oriunda da sociologia, da psicologia social e da antropologia, podendo ser aplicada no estudo de diferentes situações e tendo como foco as relações entre os atores [Wasserman e Faust 1994]. O entendimento da estrutura e da interação em uma rede social pode ser obtido através de métricas, também chamadas de propriedades de redes sociais [Wasserman e Faust 1994]. As propriedades relacionadas a um ator se baseiam nas ligações existentes entre os atores e suas relações. Dessa forma, cada ator possui um valor na rede que será considerado ao analisá-lo em relação aos demais. Essas propriedades dizem respeito à centralidade dos atores em relação à rede. Segundo Wasserman e Faust (1994), há quatro tipos de propriedades de centralidade de um ator:

- Grau do ator na rede (Degree Centrality): o ator mais central é o que possui o maior grau, ou seja, aquele que possuir maior valor dentro da rede.

- Centralidade de intermediação (Betweenness Centrality): é a capacidade que um ator tem de intermediar as comunicações entre os demais. Corresponde às interações entre dois atores não vizinhos os quais dependem de outros que se localizam entre eles. 
- Centralidade de proximidade (Closeness Centrality): é baseada na distância e representa o quão próximo um ator está de todos os outros, resultando na efetividade da comunicação e pouco esforço em se comunicar com toda a rede.

- Centralidade da informação (Information Centrality): generaliza a noção de centralidade de intermediação em todos os caminhos entre os atores, dando valores às relações dependendo do tamanho de cada caminho.

Estas propriedades permitem a análise de redes sociais e são utilizadas como base para diversas ferramentas. Além disso, as redes sociais têm sido aplicadas nas mais diversas áreas do conhecimento, para a resolução dos mais variados tipos de problemas. Recentemente, diversos mapeamentos foram realizados para outras áreas, tais como: Enfermagem [Scott et. al 2010], Computação em Nuvem [Wu e Chen 2012], Biocombustível [Xu e Boeing 2013], Gestão do Conhecimento [Sedighi e Jalalimanesh 2014] e Comportamento Informacional [González-Teruel et. al 2015]. Todos esses trabalhos serviram de base para a realização deste trabalho, principalmente no que se refere aos tipos de análises realizadas. A principal diferença em relação a esses trabalhos é que o presente mapeamento é mais abrangente pois engloba algumas análises neles presentes, além de outras obtidas a partir dessas. Apesar disso, nenhuma das análises realizadas fez uso de ferramentas utilizadas nesses trabalhos. Isso se deve principalmente ao fato de que, na maioria dos casos, essas limitam o volume de dados a serem tratados.

\section{Coleta de dados}

Inicialmente, foi realizada uma busca avançada por artigos da área de "Redes Sociais" publicados em periódicos indexados na WoS de acordo com os critérios informados na Tabela 1. O principal critério definido foi o campo "Tópico" com o conteúdo "Social Networks". Esse campo também considera os seguintes campos na sua busca: título, resumo, palavras-chave do autor e palavras-chave indexadas pela própria WoS.

O total de artigos encontrados foi 14.477, sendo 205 artigos com pelo menos um autor brasileiro. Esses artigos foram citados por 103.387 artigos e os artigos brasileiros foram citados por 975 artigos, em ambos os casos, sem levar em consideração a autocitação. A partir disso, foi possível baixar os dados de todos esses artigos no formato bibtex disponível na WoS. Dessa forma, foi feita a extração automática e os dados foram armazenados, também de forma automática, em um banco de dados. Todo esse processo, brevemente aqui descrito, foi realizado no dia 9 de abril de 2015 em poucos minutos utilizando uma linguagem de domínio específico (LDE) que está em desenvolvimento pelos autores deste trabalho. Essa LDE permite a extração automática de dados na base WoS e o armazenamento em banco de dados.

Tabela 1. Definição de critérios de busca avançada para artigos da área Redes Sociais publicados em periódicos na WoS

\begin{tabular}{ll}
\hline Campo & Critério \\
\hline Tópico (TS) & TS = (“Social Networks") \\
Tipos de Documentos & "Article" \\
Tempo estipulado & Todos os anos \\
Índices de citação & "Science Citation Index Expanded (SCI-EXPANDED)" \\
& "Social Sciences Citation Index (SSCI)" \\
\hline
\end{tabular}


As seguintes informações foram obtidas para este trabalho: artigos (id, título, tipo, ano, total de autores e total de citações), periódicos (ISSN, título) e palavras-chave dos autores. Também foram obtidos o fator de impacto (FI) de 2013 e as categorias dos periódicos no Journal Citation Reports ${ }^{\circledR}\left(\mathrm{JCR}^{\circledR}\right)$. O acesso a todas essas informações foi feito através do Portal de Periódicos da CAPES. É necessário destacar a importância do Portal de Periódicos não somente neste trabalho, mas principalmente para o avanço recente na ciência brasileira. Criado em 2000, o Portal conta hoje com um dos maiores acervos em nível mundial, assim como sua capilaridade de acesso [Almeida et. al 2010].

Os mapas apresentados neste trabalho foram gerados utilizando a ferramenta GPS Visualizer (disponível em http://www.gpsvisualizer.com). As figuras apresentadas na forma de "nuvens de palavras" (word clouds) foram geradas utilizando a ferramenta Wordle ${ }^{\mathrm{TM}}$ (disponível em http://www.wordle.net). Para gerar uma "nuvem de palavras" a ferramenta leva em consideração o número de vezes que cada palavra aparece. A visualização das redes sociais é feita na forma de grafos e para isso foi utilizada a biblioteca JUNG (disponível em http://jung.sourceforge.net). JUNG (Java Universal Network/Graph) é uma biblioteca de código aberto escrita em Java que fornece uma linguagem comum e extensível para a modelagem, análise e visualização de dados que podem ser representados como um grafo ou uma rede.

\section{Resultados e discussões}

Por meio da busca realizada verificou-se que os pesquisadores da área Redes Sociais publicaram 14.477 artigos em periódicos indexados na WoS. O Brasil com 205 artigos ocupa a décima sétima $\left(17^{\circ}\right)$ posição entre os países que mais publicaram nessa área, conforme destacado na Tabela 2. É importante notar que um mesmo artigo pode ser contabilizado por mais de um país. Isso se deve ao fato de que a WoS faz essa contagem considerando todas as afiliações informadas nos artigos [Almeida e Guimarães 2013].

O primeiro artigo da área Redes Sociais, de acordo com os periódicos indexados atualmente na WoS, foi o de H. Hamer [Hamer 1963]. Porém, o artigo mais diretamente relacionado com a área é de Barnes (1969). Também de acordo com a WoS, o artigo de Watts e Strogatz (1998) é o artigo mais citado, com 10.179 citações. O segundo artigo mais citado é de Girvan e Newman (2002), com 2.639 citações. Considerando somente os artigos com pelo menos um autor brasileiro, o primeiro artigo foi de Campos et. al (1994). O artigo mais citado é de Macinko et. al (2003), com 220 citações. Porém, nesse artigo o termo "redes sociais" aparece no resumo do artigo. Considerando o termo no título do artigo, o artigo mais citado é de Gonzalez et. al (2006), com 67 citações. O artigo mais citado contendo todos seus autores brasileiros foi o de Moreira et. al (2006), com 34 citações, sendo que esses são da Universidade Federal do Ceará.

Dos 14.447 artigos, 3.391 (23,47\%) ainda não foram citados, sendo que 700 $(20,64 \%)$ desses artigos foram publicados em 2014 ou em 2015. Os outros 11.056 artigos foram citados por 103.387 artigos, com uma média de 9,35 citações por artigo, sem considerar a autocitação. Esses artigos foram publicados por 32.801 autores, com uma média de 2,97 autores por artigo. Desse total, 2.432 (22\%) foram publicados por um único autor, $2.998(27,12 \%)$ por dois autores, $2.394(21,65 \%)$ com três autores e $3.232(29,23 \%)$ por 4 ou mais autores. 
Tabela 2. Ranking da produção científica mundial da área Redes Sociais de acordo com artigos publicados em periódicos na WoS

\begin{tabular}{|c|c|c|c|c|c|}
\hline Posição & País & Artigos & $\%$ & Citações & Posição \\
\hline $1^{\circ}$ & Estados Unidos & 6.811 & 47,05 & 81.363 & $1^{\circ}$ \\
\hline $2^{\circ}$ & Ing Ingterra & 1.606 & 11,09 & 16.479 & $2^{\circ}$ \\
\hline $3^{\circ}$ & China & 993 & 6,86 & 3.577 & $11^{\circ} \downarrow 8$ \\
\hline $4^{\circ}$ & [*] Canadá & 841 & 5,81 & 8.800 & $3^{\circ \uparrow 1}$ \\
\hline $5^{\circ}$ & Fustrália & 726 & 5,01 & 5.257 & $6^{\circ} \downarrow 1$ \\
\hline $6^{\circ}$ & $\exists$ Alemanha & 685 & 4,73 & 5.786 & $4^{\circ} \uparrow 2$ \\
\hline $7^{\circ}$ & Espanha & 676 & 4,67 & 5.084 & $7^{\circ}$ \\
\hline $8^{\circ}$ & Holanda & 610 & 4,21 & 5.390 & $5^{\circ \uparrow 3}$ \\
\hline $9^{\circ}$ & [] França & 403 & 2,78 & 3.622 & $10^{\circ} \downarrow 1$ \\
\hline $10^{\circ}$ & [】 Itália & 400 & 2,76 & 4.501 & $8^{\circ} \uparrow 2$ \\
\hline $11^{\circ}$ & 트 Suécia & 335 & 2,31 & 3.732 & $9^{\circ} \uparrow 2$ \\
\hline $12^{\circ}$ & [1] Suíça & 252 & 1,74 & 1.877 & $14^{\circ} \downarrow 2$ \\
\hline $13^{\circ}$ & :o: Coréia do Sul & 232 & 1,60 & 972 & $19^{\circ} \downarrow 6$ \\
\hline $14^{\circ}$ & $\Rightarrow$ Israel & 222 & 1,53 & 2.718 & $12^{\circ} \uparrow 2$ \\
\hline $15^{\circ}$ & - Japão & 221 & 1,53 & 1.767 & $15^{\circ}$ \\
\hline $16^{\circ}$ & $\square$ Taiwan & 209 & 1,44 & 757 & $20^{\circ} \downarrow 4$ \\
\hline $17^{\circ}$ & 8 Brasil & 205 & 1,42 & 975 & $18^{\circ} \downarrow 1$ \\
\hline $18^{\circ}$ & $\triangle$ Escócia & 204 & 1,41 & 2.255 & $13^{\circ} \uparrow 5$ \\
\hline $19^{\circ}$ & tت Finlândia & 152 & 1,05 & 1.406 & $16^{\circ} \uparrow 3$ \\
\hline $20^{\circ}$ & [』Bélgica & 147 & 1,02 & 995 & $17^{\circ} \uparrow 3$ \\
\hline
\end{tabular}

Fonte: WoS (09/04/2015)

Nos últimos anos percebe-se que o número de artigos da área Redes Sociais publicados em periódicos na WoS vem praticamente dobrando a cada 5 anos, conforme pode ser observado na Figura 1. A média de autores também está aumentando. Porém, esse aumento não é significativo comparado com a média dos períodos que é de 2,96 autores por artigo.

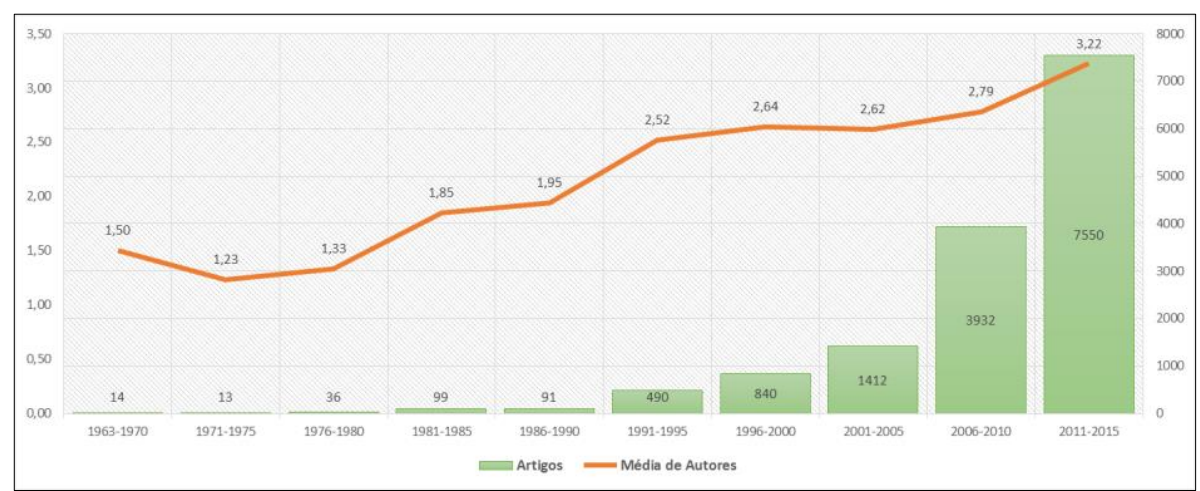

Figura 1. Número de artigos da área Redes Sociais publicados em periódicos na WoS e média de autores por períodos.

Os 14.447 artigos da área Redes Sociais foram publicados por autores de 136 países e foram citados por autores de 190 países. Os pesquisadores brasileiros, dos 205 artigos, colaboram com autores de 31 outros países (Estados Unidos: 37; Canadá: 12, Inglaterra: 12 e Alemanha: 12) e já foram citados por autores de 93 países (Estados Unidos: 298; Brasil: 167 e China: 161), sem considerar a autocitação. A Figura 2 apresenta a distribuição geográfica dos pesquisadores da área Redes Sociais que publicaram artigos em periódicos na WoS. São destacados na cor "cinza" os países em que houve menos do que 10 colaborações. 


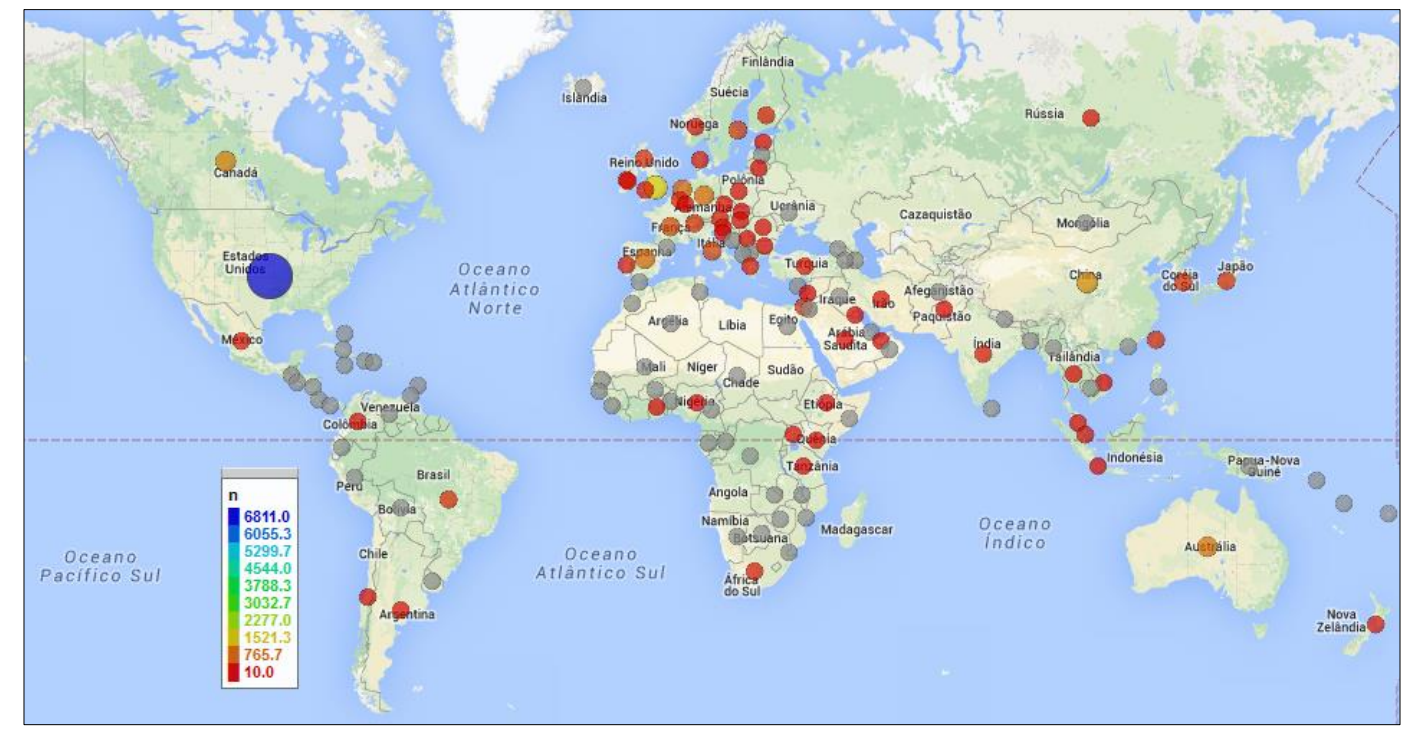

Figura 2. Distribuição geográfica da autoria da área Redes Sociais na WoS.

Com o ISSN foi obtido o FI dos periódicos de acordo com o $\mathrm{JCR}^{\circledR}$ de 2013 . A Tabela 3 apresenta a distribuição dos periódicos mais utilizados, desde 1963, para a publicação desses 14.447 artigos. Nesse período foram utilizados 2.753 periódicos diferentes com FI. Dentre esses, periódicos muito prestigiosos como a Nature (10 artigos e 14.968 citações) e a Science (15 artigos e 2.414 citações) já neles tiveram publicações. Também é apresentada a média de citações por artigos publicados em cada um dos periódicos. É possível perceber que as médias variam bastante de um periódico para outro e que nem sempre, os periódicos mais utilizados são os mais citados. Dentre os 205 artigos com pelo menos um autor brasileiro, os periódicos mais utilizados foram: Ciência \& Saúde Coletiva (17 artigos; 13 citações), Informação \& Sociedade: Estudos (9; 3), PLOS ONE $(5 ; 12)$, Physica Review E $(5 ; 173)$, Physica A $(5 ; 37)$ e Cadernos de Saúde Pública $(5 ; 17)$.

Tabela 3. Distribuição dos periódicos mais utilizados pelos autores da área Redes Sociais para publicação na WoS

\begin{tabular}{|c|c|c|c|c|c|c|}
\hline ISSN & Título do Periódico & País & Artigos (A) & Citações (C) & $\mathbf{C} / \mathbf{A}$ & FI 2013 \\
\hline $0378-8733$ & Social Networks & [a] & 303 & 5.271 & 17,40 & 2,138 \\
\hline $1932-6203$ & PLOS ONE & 㕓 & 223 & 1.266 & 5,68 & 3,534 \\
\hline 0277-9536 & Social Science \& Medicine & 㓭圆 & 198 & 5.571 & 28,14 & 2,558 \\
\hline 0378-4371 & Physica A & $\Xi$ & 197 & 1.578 & 8,01 & 1,722 \\
\hline $1539-3755$ & Physica Review E & 橉 & 162 & 4.935 & 30,46 & 2,326 \\
\hline
\end{tabular}

Fontes: WoS (09/04/2015) e $\mathrm{JCR}^{\circledR}(2013)$

Analisando os periódicos mais utilizados ao longo desse período, percebe-se, para os dois últimos períodos, que o número de artigos aumentou de forma considerável, principalmente no periódico PLOS ONE, que no período de 2006 a 2010 teve 18, passando para 205 no período seguinte, conforme pode ser observado na Figura 3. Nesses periódicos foram publicados 1.083 artigos, o que corresponde a 7,50\% do total da área Redes Sociais. E somente no último período foram publicados $561(51,80 \%)$ artigos, o que demonstra que esses periódicos ainda são muito utilizados. 


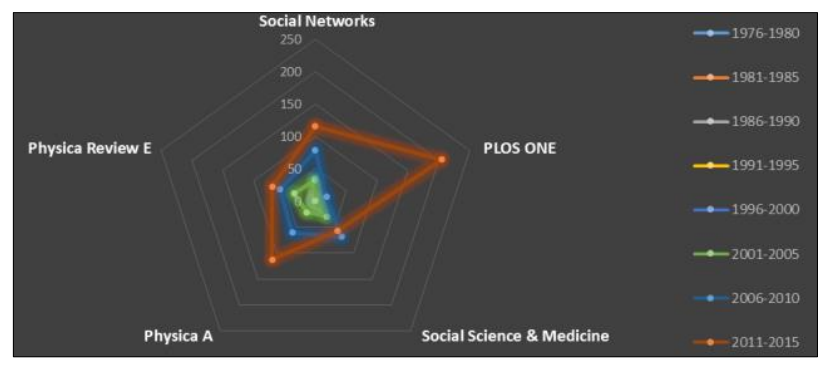

Figura 3. Análise temporal dos periódicos mais utilizados pelos autores da área Redes Sociais para publicação na WoS.

Do total de 14.477 artigos, em $14.471(99,96 \%)$ foi possível obter pelo menos uma categoria dos periódicos de acordo com a classificação na WoS. A Figura 4 apresenta as categorias mais utilizadas pelos autores da área Redes Sociais. As principais são: "Sociology" (1.480; 10,24\%), "Public Environmental Occupational Health" (1.223; 8,47\%) e "Computer Science, Information Systems" (1.018; 7,05\%). Percebe-se, então, que a área Redes Sociais é transdisciplinar, possuindo relação direta com as palavraschave apresentadas da Figura 5.

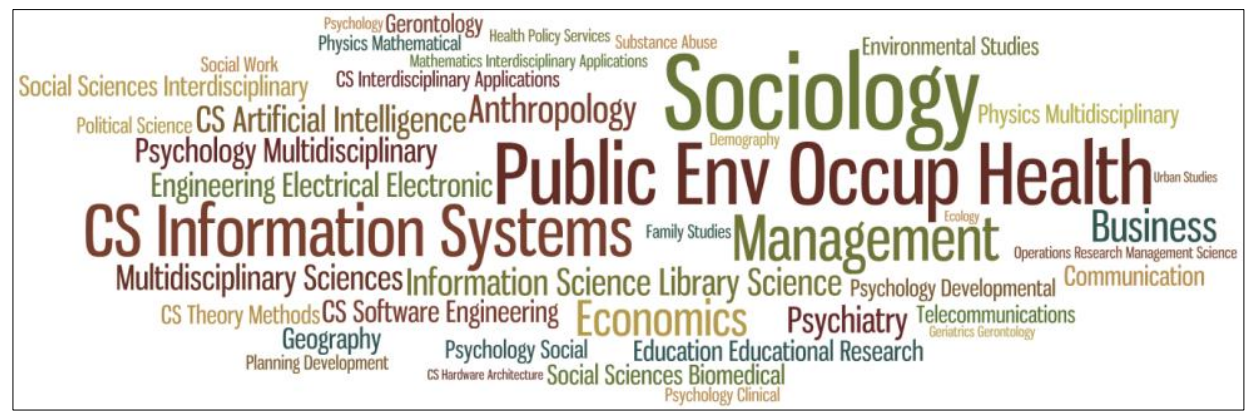

Figura 4. Categorias dos periódicos mais utilizados pelos autores da área Redes Sociais para publicação na WoS.

É importante lembrar que esses artigos foram identificados considerando diversos campos na sua busca na WoS. Dessa forma, se no resumo do artigo foi informado o termo "Social Networks" o mesmo foi considerado. No caso das categorias, o que pode ocorrer é que categorias que talvez não sejam consideradas tão relacionadas com a área se destaquem. Por exemplo, a categoria "Public Environmental Occupational Health" aparentemente não tem relação com a área. Entretanto, verificando o termo "Social Networks" no título dos artigos, percebe-se que essa categoria tem resultado semelhante ao da categoria "Sociology". Na categoria "Public Environmental Occupational Health" o termo aparece em 294 (24,04\%) artigos e na categoria "Sociology" em 373 (25,20\%) artigos. Na categoria "Computer Science, Information Systems" o termo aparece em 371 $(36,44 \%)$ artigos. Considerando as palavras-chave dos autores, o resultado é muito semelhante: "Sociology" (368; 24,86\%), "Public Environmental Occupational Health" (293; 23,96\%) e “Computer Science, Information Systems" (305; 29,96\%).

Também foi realizado uma análise temporal das palavras-chave mais utilizadas pelos autores da área Redes Sociais, conforme ilustrado na Figura 5. Essa análise considerou quatro períodos de 5 anos, iniciando em 1996 que foi o ano que a WoS começou a indexar as palavras-chave dos artigos. Para montar essa "nuvem de palavras", foram obtidas as 20 palavras-chave mais utilizadas em cada um dos períodos e uma cor foi associada. As palavras-chave "Social Network" e "Social Networks" não foram consideradas nessa análise. 


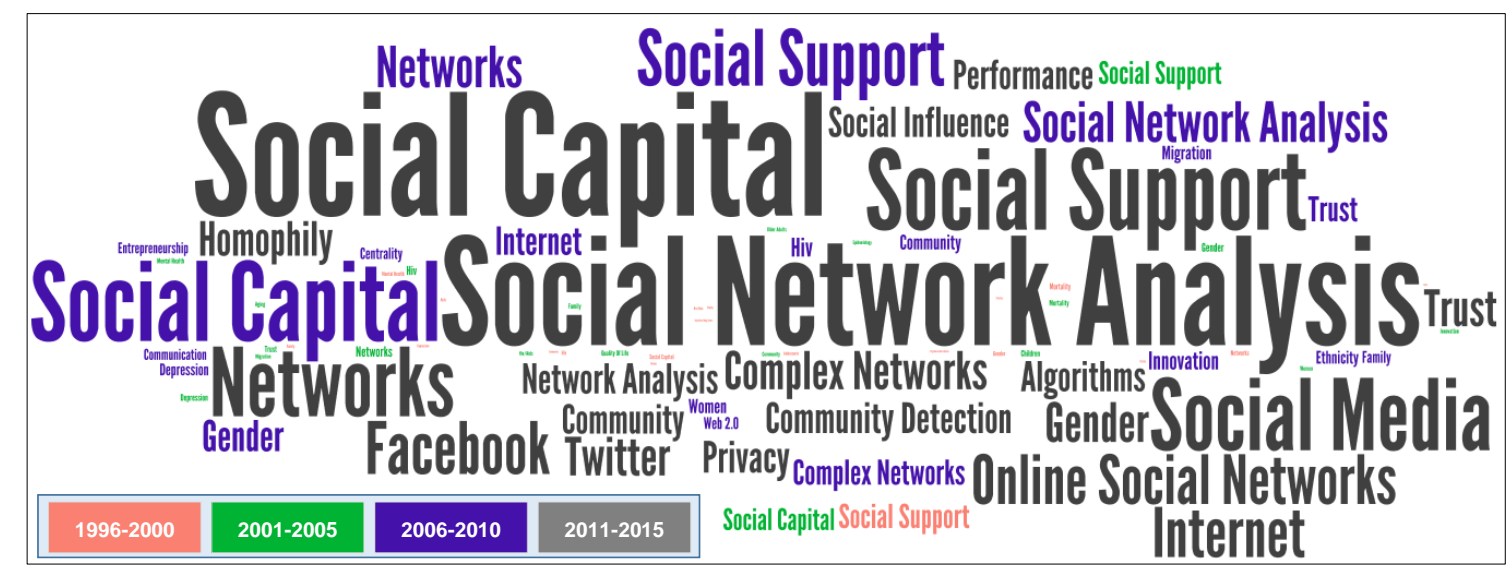

Figura 5. Análise temporal das palavras-chave mais utilizadas pelos autores da área Redes Sociais que publicaram em periódicos na WoS.

As palavras-chave "Social Capital" e "Social Support" aparecem em todos os períodos e a palavra-chave "Social Network Analysis" foi bastante utilizada nos dois últimos períodos. É importante destacar que esse termo (ou variações) foi utilizado em $362(2,51 \%)$ artigos e 258 (71,27\%) desses foram publicados depois de 2010. Os autores dos artigos dessa área são de 58 países e já foram citados por autores de 89 países.

A rede de coautoria da área Redes Sociais entre os 20 países que mais publicaram em periódicos na WoS é apresentada na Figura 6. Para cada país foram obtidos os 20 (máximo) países com quem os autores desses artigos mais colaboraram. Dessa forma, foram encontrados 57 países com 270 coautorias entre eles. Entretanto, o grafo conectado apresentado na Figura 6 contém apenas 43 países (vértices) e 256 coautorias (arestas). Isso é porque todos os vértices com grau 1 foram removidos. Em todos os grafos apresentados neste trabalho, a cor dos vértices é definida pelo grau de cada vértice e a cor das arestas é definida de acordo com o número de coautorias. O tamanho dos vértices representa o número de artigos e a largura e intensidade das cores das arestas representam o quão intensa é uma relação. Percebe-se que as principais relações ocorrem entre os Estados Unidos e a China (256) e entre os Estados Unidos e a Inglaterra (208).

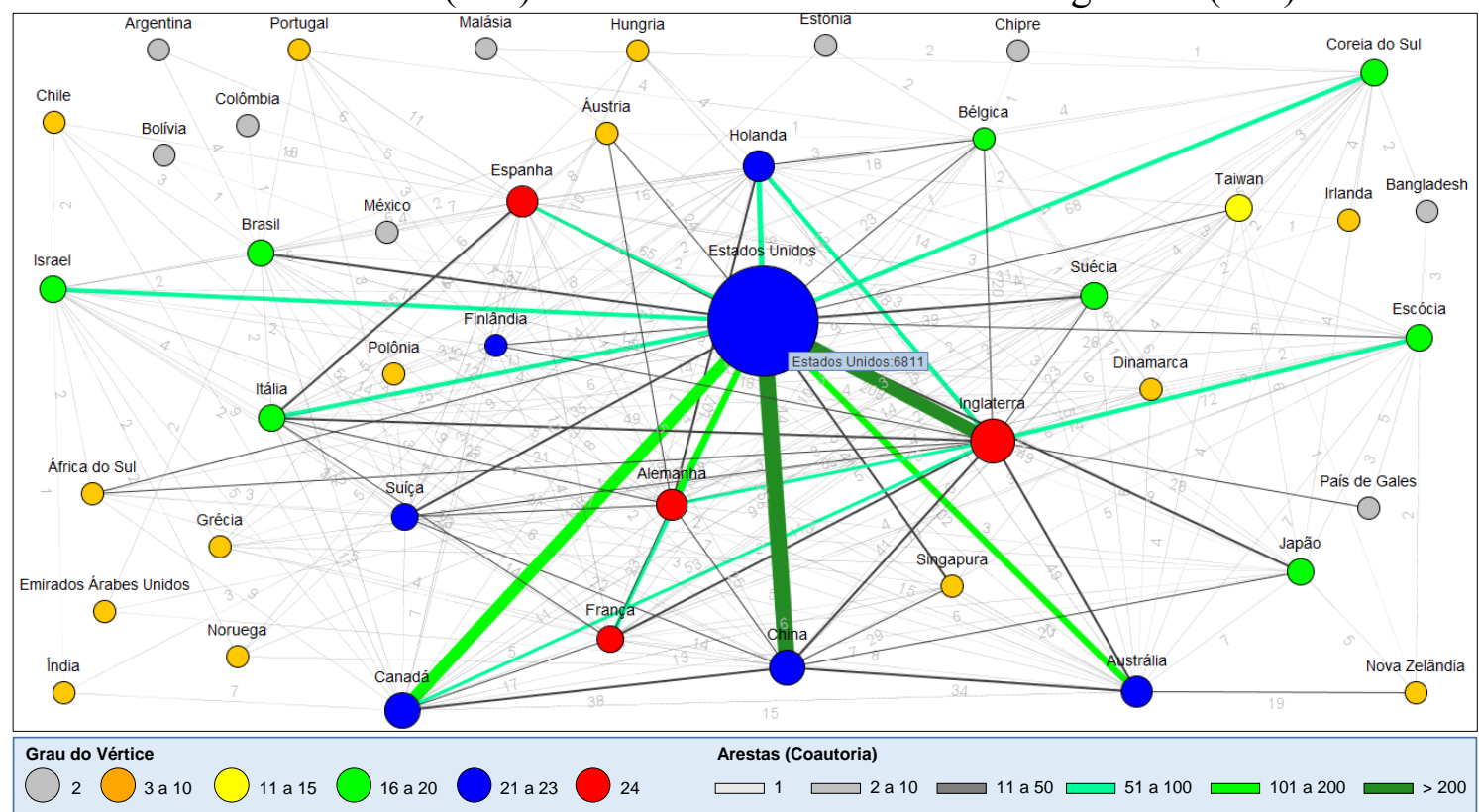

Figura 6. Rede de coautoria da área Redes Sociais entre os países que mais publicaram em periódicos na WoS. 
A partir do grafo gerado na Figura 6, foi possível calcular as medidas de centralidade entre os países, conforme apresentado na Tabela 4, sendo que apenas os países com grau igual ou superior a 20 foram considerados. Percebe-se que, apesar dos Estados Unidos serem o país que mais publicou artigos na área Redes Sociais, o país que mais se destaca é a Espanha, pois é central em todas as medidas. Percebe-se também que além da Espanha, Inglaterra, Alemanha e França são países que se destacam na área Redes Sociais considerando as medidas de centralidade. Entretanto, apenas a Inglaterra se destaca quando é considerado o número de artigos ou de citações. O Brasil também merece destaque sendo o segundo país mais importante ao se considerar a medida de centralidade de intermediação.

Tabela 4. Medidas de Centralidade da área Redes Sociais entre os países que mais publicaram em periódicos na WoS

\begin{tabular}{|c|c|c|c|c|}
\hline País & Grau & Intermediação & Proximidade & Informação \\
\hline Espanha & 24 & $84,178^{\left(1^{\circ}\right)}$ & $0,700^{\left(1^{\circ}\right)}$ & $0,017^{\left(1^{\circ}\right)}$ \\
\hline 果 Inglaterra & 24 & $43,797^{\left(8^{\circ}\right)}$ & $0,700^{\left(2^{\circ}\right)}$ & $0,017^{\left(2^{\circ}\right)}$ \\
\hline$\exists$ Alemanha & 24 & $31,291^{\left(12^{\circ}\right)}$ & $0,700^{\left(3^{\circ}\right)}$ & $0,017^{\left(3^{\circ}\right)}$ \\
\hline [] França & 24 & $23,963^{\left(14^{\circ}\right)}$ & $0,700^{\left(4^{\circ}\right)}$ & $0,017^{\left(4^{\circ}\right)}$ \\
\hline Austrália & 23 & $37,443^{\left(9^{\circ}\right)}$ & $0,689^{\left(5^{\circ}\right)}$ & $0,016\left(^{\circ}\right)$ \\
\hline$\llbracket * \rrbracket$ Canadá & 23 & $33,614^{\left(11^{\circ}\right)}$ & $0,689^{\left(6^{\circ}\right)}$ & $0,016^{\left(6^{\circ}\right)}$ \\
\hline 泿青 Estados Unidos & 22 & $16,202^{\left(17^{\circ}\right)}$ & $0,677^{\left(7^{\circ}\right)}$ & $0,016^{\left(7^{\circ}\right)}$ \\
\hline I Finlândia & 21 & $58,661^{\left(4^{\circ}\right)}$ & $0,656^{\left(10^{\circ}\right)}$ & $0,016^{\left(10^{\circ}\right)}$ \\
\hline [1] Suíça & 21 & $37,175^{\left(10^{\circ}\right)}$ & $0,646^{\left(12^{\circ}\right)}$ & $0,015^{\left(12^{\circ}\right)}$ \\
\hline Holanda & 21 & $18,344^{\left(16^{\circ}\right)}$ & $0,667^{\left(8^{\circ}\right)}$ & $0,016^{\left(8^{\circ}\right)}$ \\
\hline China & 21 & $15,900^{\left(18^{\circ}\right)}$ & $0,667^{\left(9^{\circ}\right)}$ & $0,016^{\left(9^{\circ}\right)}$ \\
\hline Brasil & 20 & $71,910\left(^{\circ}\right)$ & $0,646^{\left(13^{\circ}\right)}$ & $0,015^{\left(13^{\circ}\right)}$ \\
\hline E Suécia & 20 & $29,968^{\left(13^{\circ}\right)}$ & $0,656^{\left(11^{\circ}\right)}$ & $0,016^{\left(11^{\circ}\right)}$ \\
\hline 【1 Itália & 20 & $13,734^{\left(19^{\circ}\right)}$ & $0,646^{\left(14^{\circ}\right)}$ & $0,015^{\left(14^{\circ}\right)}$ \\
\hline
\end{tabular}

De forma análoga aos países, foi gerada a rede de coautoria da área Redes Sociais para os 20 autores que mais publicaram em periódicos na WoS identificando os 20 (máximo) autores com quem esses mais colaboraram. Foram encontrados 362 autores com 376 coautorias entre eles, conforme ilustrado na Figura 7. O grafo gerado contém 11 componentes (clusters), sendo que o maior deles possui 100 autores, destacado na Figura 7 em vermelho. Os demais componentes estão delimitados por linhas pretas pontilhadas. Os outros componentes variam de 11 a 50, tendo como moda o valor 21 . As principais relações de coautoria ocorrem entre os seguintes pares de autores: "Christakis NA" e "Fowler JH" (20); "Robins G" e "Pattison P" (15); "Wang L" e "Fu F" (11).

Para identificar os principais componentes dos clusters de coautoria da área Redes Sociais, foram removidos recursivamente todos os vértices com grau 1. Com isso, foram identificados 2 componentes, o maior com 20 autores e o outro com 13 autores, conforme ilustrado na Figura 8. Nesse grafo, com 33 vértices e 56 arestas, é possível identificar as principais relações destacadas anteriormente. É possível visualizar também a importância do autor "Wang L", que é elo de ligação entre dois grupos de pesquisadores. Além disso, percebe-se que o autor "Latkin CA" foi quem mais publicou na área. De acordo com a WoS, esse autor publicou 44 artigos. Porém, foi identificado que esse autor e o autor 
"Latkin C" (25 artigos) são o mesmo pesquisador e, portanto, foram considerados como um único pesquisador e com 69 artigos. A WoS considera-os como dois pesquisadores dado que a afiliação informada não ser a mesma. Isso deve acontecer também com outros pesquisadores.

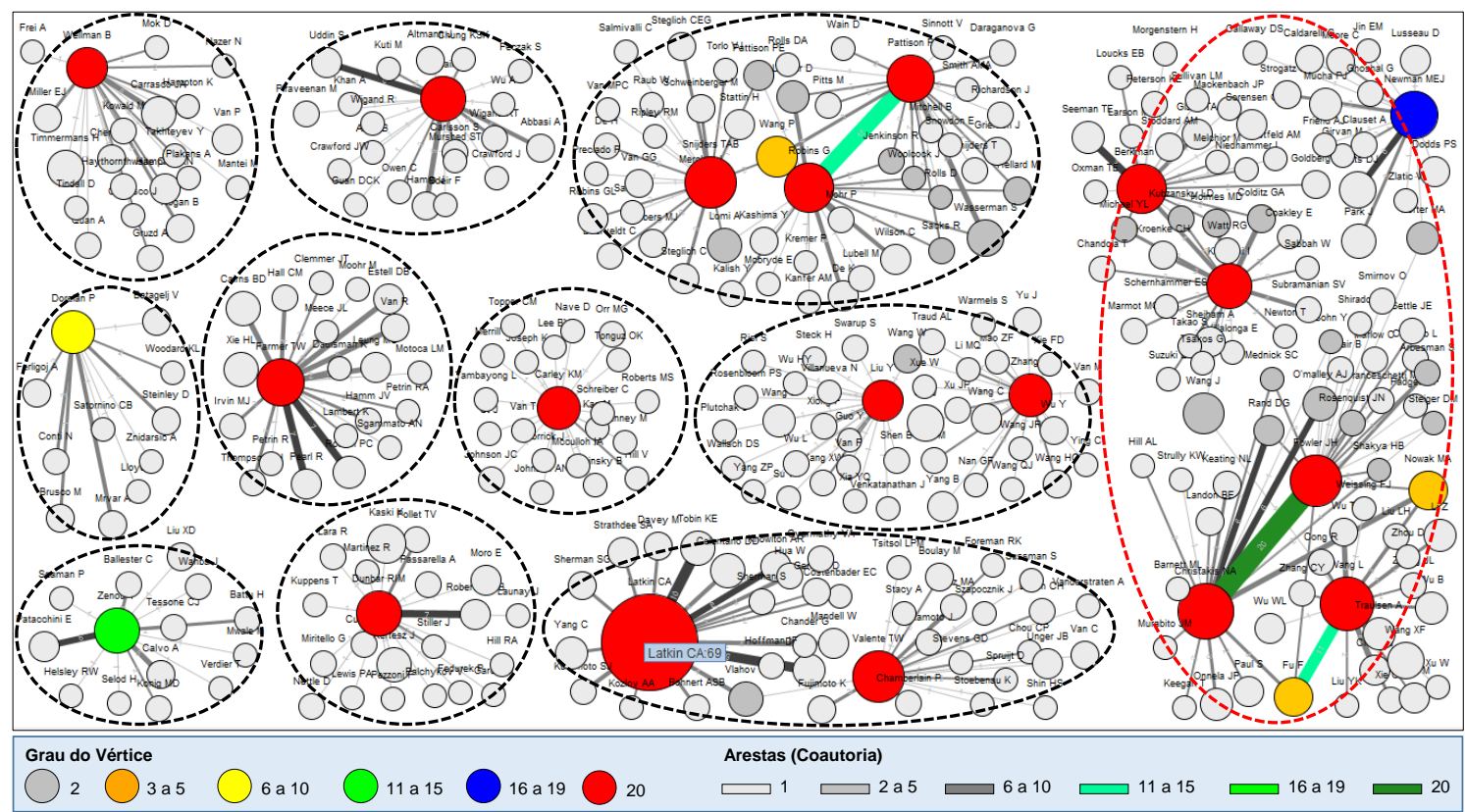

Figura 7. Rede de coautoria da área Redes Sociais entre os autores que mais publicaram em periódicos na WoS.

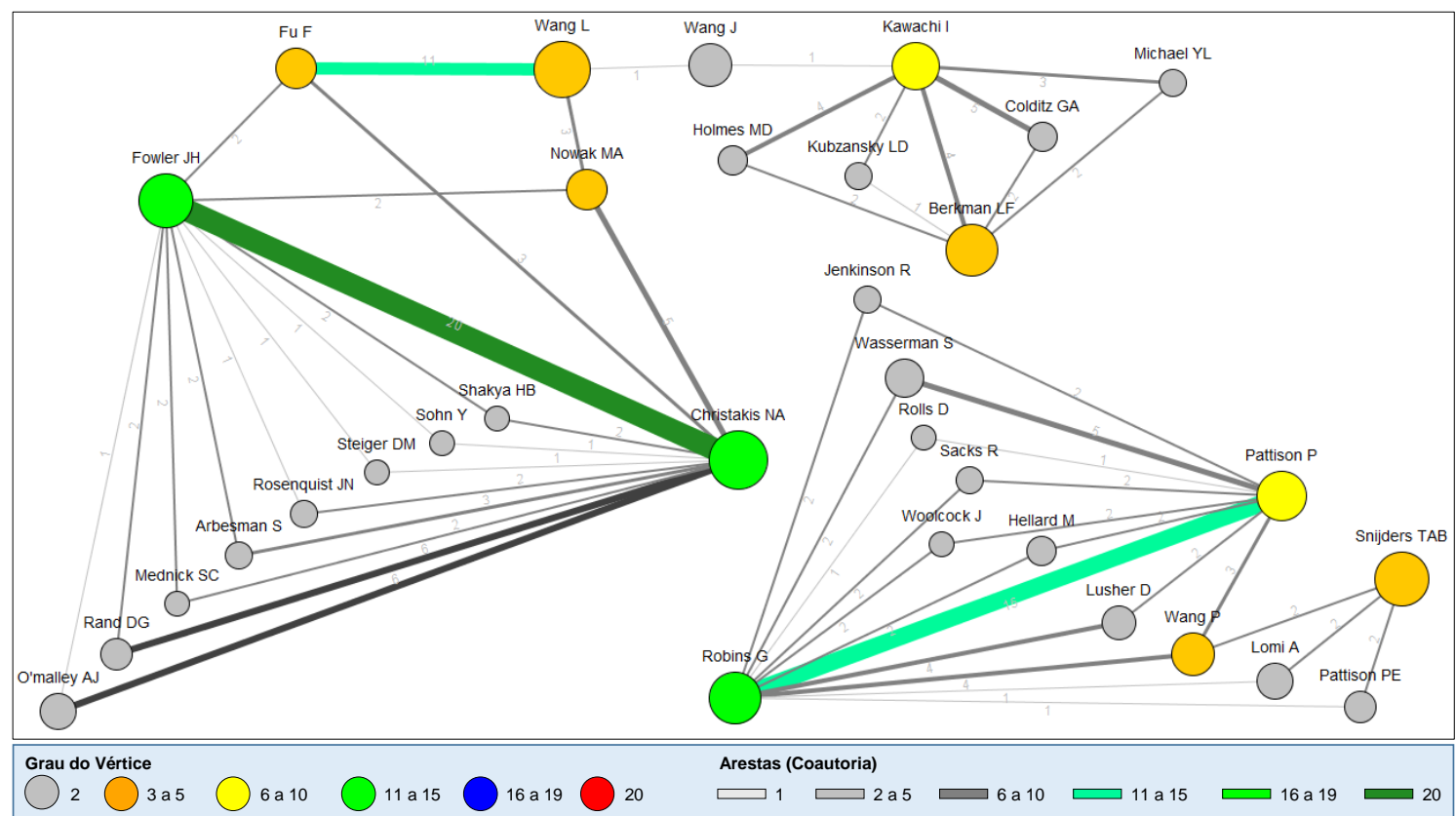

Figura 8. Componentes dos clusters de coautoria da área Redes Sociais na WoS.

A partir desse grafo, também foi possível calcular as medidas de centralidade entre os autores, conforme apresentado na Tabela 5. No caso de empate foi considerado o grau dos autores. Percebe-se que os dois primeiros autores possuem exatamente os mesmos valores para todas as medidas de centralidade e nos grafos das Figuras 7 e 8 ficam 
evidentes as relações de coautoria entre eles. É importante lembrar que esses dois autores são a principal relação de coautoria. Aqui também fica claro que nem sempre os autores que mais publicam são os que mais se destacam considerando as medidas de centralidade.

Tabela 5. Medidas de Centralidade da área Redes Sociais entre os autores que mais publicaram em periódicos na WoS

\begin{tabular}{lccccc}
\hline Autor & Artigos & Grau & Intermediação & Proximidade & Informação \\
\hline Christakis NA & $31^{\left(2^{\circ}\right)}$ & 11 & $54,333^{\left(4^{\circ}\right)}$ & $0,422^{\left(14^{\circ}\right)}$ & $0,022^{\left(14^{\circ}\right)}$ \\
Fowler JH & $27^{\left(6^{\circ}\right)}$ & 11 & $54,333^{\left(5^{\circ}\right)}$ & $0,422^{\left(15^{\circ}\right)}$ & $0,022^{\left(15^{\circ}\right)}$ \\
Robins G & $26^{\left(7^{\circ}\right)}$ & 11 & $36,750^{\left(8^{\circ}\right)}$ & $0,923^{\left(1^{\circ}\right)}$ & $0,077^{\left(1^{\circ}\right)}$ \\
Pattison P & $23^{\left(10^{\circ}\right)}$ & 9 & $15,750^{\left(9^{\circ}\right)}$ & $0,800^{\left(2^{\circ}\right)}$ & $0,067^{\left(2^{\circ}\right)}$ \\
Kawachi I & $22^{\left(12^{\circ}\right)}$ & 6 & $73,000^{\left(3^{\circ}\right)}$ & $0,306^{\left(20^{\circ}\right)}$ & $0,016^{\left(20^{\circ}\right)}$ \\
Berkman LF & $25^{\left(8^{\circ}\right)}$ & 5 & $3,000^{\left(11^{\circ}\right)}$ & $0,250^{\left(29^{\circ}\right)}$ & $0,013^{\left(29^{\circ}\right)}$ \\
Wang L & $29^{\left(3^{\circ}\right)}$ & 3 & $84,333^{\left(1^{\circ}\right)}$ & $0,396^{\left(18^{\circ}\right)}$ & $0,021^{\left(18^{\circ}\right)}$ \\
Nowak MA & $15^{\left(29^{\circ}\right)}$ & 3 & $40,000^{\left(6^{\circ}\right)}$ & $0,413^{\left(16^{\circ}\right)}$ & $0,022^{\left(16^{\circ}\right)}$ \\
Fu F & $15^{\left(38^{\circ}\right)}$ & 3 & $40,000^{\left(7^{\circ}\right)}$ & $0,413^{\left(17^{\circ}\right)}$ & $0,022^{\left(17^{\circ}\right)}$ \\
Wang P & $17^{\left(22^{\circ}\right)}$ & 3 & $4,833^{\left(10^{\circ}\right)}$ & $0,571^{\left(3^{\circ}\right)}$ & $0,048^{\left(3^{\circ}\right)}$ \\
\hline
\end{tabular}

\section{Conclusões}

Este trabalho evidencia o crescimento recente e significativo da área Redes Sociais, visto que o número de artigos publicados em periódicos na WoS praticamente dobra a cada 5 anos. Além disso, essa área possui autores de 136 países e já foi citada por autores de 190 países. Outro resultado interessante e até mesmo inesperado, foi o fato de que os Estados Unidos, embora seja o país com maior publicação nessa área, não seja aquele que mais se destaca quando as medidas de centralidade são consideradas. Nesse caso, a Espanha é o país mais "central" e os outros 3 países da Europa (Inglaterra, Alemanha e França) também se destacam. Outro país que possui destaque é o Brasil, sendo o segundo em termos da centralidade de intermediação.

Uma das contribuições deste trabalho é o de poder auxiliar a realização de estudos em outras áreas do conhecimento. O mapeamento da produção científica permite mostrar a evolução da pesquisa na área. Além disso, conhecer o perfil de uma área também permite definir estratégias de atuação e indicar tendências das pesquisas sendo realizadas. Este trabalho também mostrou que é possível realizar estudos mais abrangentes, envolvendo diversos tipos de análises e uma grande quantidade de dados, pois a coleta, extração e indexação desses dados são feitas de forma automática, bem como a maioria das análises apresentadas neste trabalho.

\section{Agradecimentos}

Os autores agradecem o apoio financeiro da CAPES e CNPq. Os autores agradecem aos revisores anônimos por suas importantes contribuições para a melhoria deste manuscrito.

\section{Referências}

Almeida, E.C.E., Guimarães, J.A., Alves, I.T.G. (2010). Dez anos do Portal de Periódicos da CAPES: histórico, evolução e utilização. Revista Brasileira de Pós-Graduação (RBPG), 7(13):218-246. 
Almeida, E.C.E., Guimarães, J.A. (2013). Brazil's growing production of scientific articles - how are doing with review articles and other qualitative indicators? Scientometrics, 97(2):287-315.

Barnes, J.A. (1969). Graph Theory and Social Networks: a technical comment on Connectedness and Connectivity. Sociology, 3(2):215-232.

Borgatti, S.P., Mehra, A., Brass, D.J., Labianca, G. (2009). Network Analysis in the Social Sciences. Science, 323(5916):892-895.

Campos, R., Raffaelli, M., Ude, W., Greco, M., Ruff, A. et al. (1994). Social Networks and daily activities of street youth in Belo-Horizonte, Brasil. Child Development, 65(2):319-330.

Girvan, M., Newman, M.E.J. (2002). Community structure in social and biological networks. Proceedings of the National Academy of Sciences of the United States of America, 99(12):7821-7826.

Gonzalez, M.C., Lind, P.G., Herrmann, H.J. (2006). System of mobile agents to model social networks. Physical Review Letters, 96(8):088702.

González-Teruel, A., Gonzáles-Alcaide, G., Barrios, M., Abad-García, M.-F. (2015). Mapping recent information behavior research: an analysis of co-authorship and cocitation networks. Scientometrics, 103(2):687-705.

Hamer, H. (1963). Influence of Small Social Networks as factors on mental-hospital admission. Human Organization, 22(4):243-251.

Macinko, J., Starfield, B., Shi, LY. (2003). The contribution of primary care systems to health outcomes within Organization for Economic Cooperation and Development (OECD) countries, 1970-1998. Health Services Research, 38(3):831-865.

Milgram, S. (1967). The small world problem. Psychology Today, 1(1):61-67.

Moreira, A.A., Paula, D.R., Costa, R.N., Andrade, J.S. (2006). Competitive cluster growth in complex networks. Physical Review E, 73(6):065101.

Moreno, J.L. (1934). Who Shall Survive? A new approach to the problem of human interrelations, Nervous and Mental Disease Publishing Co.

Newman. M., Barabási, A.-L., Watts, D.J. (2006). The Structure and Dynamics of Networks, Princeton University Press.

Scott, S.D., Profetto-McGrath, J., Estabrooks, C.A., Winther, C. Wallin, L., Lavis, J.N. (2010). Mapping the Knowledge Utilization Field in Nursing from 1945 to 2004: A Bibliometric Analysis. Worldviews on Evidence-Based Nursing, 7(4):226-237.

Sedighi, M., Jalalimanesh, A. (2014). Mapping research trends in the field of Knowledge Management. Malaysian Journal of Library \& Information Science, 19(1):71-85.

Xu Y., Boeing, W.J. (2013). Mapping biofuel field: A bibliometric evaluation of research output. Renewable and Sustainable Energy Reviews, 28: 82-91.

Wasserman, S., Faust, K. (1994). Social network analysis: methods and applications (Structural Analysis in the Social Sciences), Cambridge University Press.

Watts, D.J., Strogatz, S.H. (1998). Collective dynamics of 'small-world' networks. Nature, 393(6684):440-442.

$\mathrm{Wu}$, J., Chen, Q. (2012). Mapping the emerging field of cloud computing: insights from a visualization analysis. In: 2012 IEEE Conference on Systems, Man, and Cybernetics (SMC), pages 1794-1799, Seoul, Korea. 